\title{
Minimum Symbol Error Probability MIMO Design under the Per-Antenna Power Constraint
}

\author{
Enoch Lu and I.-Tai Lu \\ Department of Electrical and Computer Engineering, Polytechnic Institute of NYU, 6 MetroTech Center, Brooklyn, NY 11201, USA \\ Correspondence should be addressed to Enoch Lu, enoch.school@gmail.com
}

Received 21 October 2011; Revised 26 January 2012; Accepted 31 January 2012

Academic Editor: D. Laurenson

Copyright () 2012 E. Lu and I.-T. Lu. This is an open access article distributed under the Creative Commons Attribution License, which permits unrestricted use, distribution, and reproduction in any medium, provided the original work is properly cited.

Approximate minimum symbol error probability transceiver design of single user MIMO systems under the practical per-antenna power constraint is considered. The upper bound of a lower bound on the minimum distance between the symbol hypotheses is established. Necessary conditions and structures of the transmit covariance matrix for reaching the upper bound are discussed. Three numerical approaches (rank zero, rank one, and permutation) for obtaining the optimum precoder are proposed. When the upper bound is reached, the resulting design is optimum. When the upper bound is not reached, a numerical fix is used. The approach is very simple and can be of practical use.

\section{Introduction}

Since multiple-input and multiple-output (MIMO) is a very promising technology for mitigating the spectrum scarcity problem, many MIMO transceiver designs have been published recently. The designs have been based on a variety of criteria, for example, maximum capacity, minimum mean square error (MMSE) and minimum bit error rate (Min BER). Considered in this paper is the minimum symbol error probability transceiver design subject to the per-antenna power constraint. This is due to a number of reasons. Firstly, the minimum symbol error probability criterion is directly related to the system performance. Secondly, the per-antenna power constraint is more practical than the commonly used total power constraint in MIMO systems (because each antenna has its own power amplifier and each power amplifier has a limited dynamic range). Lastly, both criterion and metric are difficult to tackle.

Minimum symbol error probability and the related Min BER design problems have been formulated in various different ways (e.g., [1-7]). For example, [1] performs its minimum symbol error probability design by maximizing a lower bound for the minimum distance of the symbol hypotheses. As the metric is a lower bound, the design is approximate in nature. However, it is also thus independent of the symbol alphabet. Their lower bound is the minimum eigenvalue of a positive definite system matrix thus making the problem a max min eigenvalue one. For the sum power constraint, their optimum design is neatly obtained by making all eigenvalues equal.

In this paper, we follow the formulation in [1] because it involves interesting and challenging signal processing issues. As already mentioned, the constraint here however is the per-antenna one. The upper bound of the cost function is established using the per-antenna power constraint and the special structure of a system matrix (which involves the precoder matrix, noise covariance matrix, and channel matrix). The necessary conditions and structures of the transmit covariance matrix for reaching the upper bound are discussed in detail. Three numerical approaches (rank zero, rank one, and permutation) for obtaining the optimum precoder are proposed. When the upper bound is reached, the resulting design is optimum. When the upper bound is not reached, a numerical fix is used. Extensive numerical studies have been performed to assess the performance of the proposed methodology. Although the upper bound is not reached in most cases, good performances in mutual information and signal to interference plus noise ratio (SINR) are achieved. Moreover, the approach is very simple and can be of practical use. 
Notations: boldface letters denote either vectors (lower case) or matrices (upper case). $\mathbf{A}^{T}, \mathbf{A}^{*}, \mathbf{A}^{-1}, \operatorname{tr}(\mathbf{A}), E(\mathbf{A})$, $\lambda_{\max }(\mathbf{A})$, and $\lambda_{\min }(\mathbf{A})$ stand for the transpose, conjugate transpose, inverse, trace, expectation, maximum eigenvalue, and minimum eigenvalue of $\mathbf{A}$, respectively. $\lambda_{i}(\mathbf{A})$ stands for the $i$ th eigenvalue of $\mathbf{A}$ when its eigenvalues are arranged in increasing order. $\mathbf{I}_{r}$ is the $r \times r$ identity matrix. $\mathbf{0}$ is the zero matrix of appropriate dimension. $\mathbf{e}_{i}$ denotes the $i$ th column of the identity matrix (the size of which will be clear from the context). $\operatorname{diag}(\mathbf{a})$ denotes the diagonal matrix with $\mathbf{a}$ on the main diagonal. On the other hand, if $\mathbf{A}$ is a square matrix, $\operatorname{diag}(\mathbf{A})$ is the main diagonal of $\mathbf{A} . \mathbf{A}>\mathbf{B}$ and $\mathbf{A} \geq \mathbf{B}$ mean that $\mathbf{A}-\mathbf{B}$ is positive definite and positive semidefinite, respectively. $\mathbf{A}^{\circ} \mathbf{B}$ denotes the elementwise product of $\mathbf{A}$ and B. $\max (a, b)$ means the maximum of real numbers $a$ and $b$. $\mathrm{CN}(0, \mathbf{R})$ denotes a zero-mean circularly symmetric complex normal random vector with covariance matrix $\mathbf{R}$.

\section{Background, Problem, and Overview of Design}

The received signal of the considered MIMO system is $\mathbf{y}=$ HFs $+\mathbf{n}$ where $\mathbf{H}$ is the full rank $n \times n$ channel matrix, $\mathbf{F}$ is the full rank $n \times n$ precoder, $\mathbf{s}$ is the $n \times 1$ data vector, and $\mathbf{n}$ is the $n \times 1$ received noise vector. The full rank $n \times n$ decoder $\mathrm{G}$ is applied to $\mathbf{y}$ to yield Gy. For convenience and without loss of generality, let the source covariance matrix $E\left(\mathbf{s s}^{*}\right)=\mathbf{I}$ and the noise covariance matrix $E\left(\mathbf{n n}^{*}\right)=\mathbf{R}_{\mathbf{n n}}>\mathbf{0}$. As the transmitter's power must be constrained, the precoder $\mathbf{F}$ is required to satisfy the per-antenna power constraint

$$
\mathbf{e}_{i}^{*}\left(\mathrm{FF}^{*}\right) \mathbf{e}_{i} \leq d_{i}, \quad \forall i
$$

Here, $d_{i}>0, \forall i$. Note that (1) also results in the average total power being upper bounded by $P=\sum_{i=1}^{n} d_{i}$. Let $S$ denote the set of all feasible F's.

2.1. Problem Formulation. The goal of this paper is to design $\mathbf{F}$ and $\mathbf{G}$ to approximately minimize the probability of error in an alphabet independent manner. To accomplish this, we will begin as in [1]. Define $\mathbf{A} \triangleq \mathbf{H}^{*} \mathbf{R}_{\mathrm{nn}}^{-1} \mathbf{H}$ and

$$
\mathbf{\Psi}(\mathrm{F}, \mathrm{G}) \triangleq \mathrm{F}^{*} \mathbf{H}^{*} \mathbf{G}^{*}\left(\mathrm{GR}_{\mathrm{nn}} \mathbf{G}^{*}\right)^{-1} \mathrm{GHF} .
$$

$\lambda_{\min }(\Psi(\mathbf{F}, \mathbf{G}))$ actually lower bounds the minimum distance between the symbol hypotheses. In addition,

$$
\Psi(\mathrm{F}, \mathrm{G}) \leq \mathrm{F}^{*} \mathrm{AF}
$$

with equality when $\mathbf{G}=\mathbf{F}^{*} \mathbf{H}^{*}\left(\mathbf{H F F} \mathbf{H}^{*} \mathbf{H}^{*}+\mathbf{R}_{\mathbf{n n}}\right)^{-1}$, that is, the MMSE decoder is used. Thus, we will choose $\mathbf{G}=\mathbf{F}^{*} \mathbf{H}^{*}\left(\mathbf{H F F}^{*} \mathbf{H}^{*}+\mathbf{R}_{\mathbf{n n}}\right)^{-1}$ and design the precoder $\mathbf{F}$ according to the optimization problem:

$$
\max _{\mathbf{F} \in S} \mu(\mathbf{F}), \quad \mu(\mathbf{F}) \triangleq \lambda_{\min }\left(\mathbf{F}^{*} \mathbf{A F}\right) .
$$

Note that if the equality average total power constraint $\operatorname{tr}\left(\mathbf{F F}^{*}\right)=P$ was used instead, the solution to (4) would be given by [1]. That is,

$$
\mathbf{V} \boldsymbol{\Lambda}^{-1 / 2}\left(\frac{P}{\operatorname{tr}\left(\boldsymbol{\Lambda}^{-1}\right)}\right)^{1 / 2}=\underset{\operatorname{tr}\left(\mathbf{F F}^{*}\right)=P, \mathbf{F} \in \mathbb{C}^{n \times n}}{\operatorname{argmax}} \mu(\mathbf{F}),
$$

where the unitary matrix $\mathbf{V}$ and the diagonal matrix $\boldsymbol{\Lambda}$ are obtained from the eigenvalue decomposition of $\mathbf{A}=\mathbf{V} \boldsymbol{\Lambda} \mathbf{V}^{*}$ (eigenvalues in descending order).

2.2. Overview of Design of $\mathbf{F}$. The optimization problem, (4), is very complicated. Though not mentioned in Section 2.1, we desire a low complexity algorithm to design $\mathbf{F}$. We will thus take some simplifications.

Before detailing the simplifications and the algorithm, we will first need some analysis on the cost function of (4): since $\mathbf{F}^{*} \mathbf{A F}$ and $\mathbf{A F F}^{*}$ have the same eigenvalues, $\mathbf{A F F}^{*} \mathbf{z}=\mu(\mathbf{F}) \mathbf{z}$ where $\mathbf{z}$ is an eigenvector of $\mathbf{A F F}{ }^{*}$ generated by the minimum eigenvalue $\mu(\mathbf{F})$. Noting that $\mathbf{A}>\mathbf{0}$,

$$
\mu(\mathbf{F})=\frac{\mathbf{z}^{*} \mathbf{F F}^{*} \mathbf{z}}{\mathbf{z}^{*} \mathbf{A}^{-1} \mathbf{z}} \leq \frac{\mathbf{x}^{*} \mathbf{F F}^{*} \mathbf{x}}{\mathbf{x}^{*} \mathbf{A}^{-1} \mathbf{x}},
$$

where $\mathbf{x}$ is any nonzero $n \times 1$ vector. Define $\mathbf{B}=\left[b_{i j}\right] \triangleq$ $\mathbf{A}^{-1}$ for convenience. Plugging in $\mathbf{e}_{i}$ for $\mathbf{x}$ in (6) and using the constraint (1), it is then clear that, for all $\mathbf{F} \in S$,

$$
\mu(\mathbf{F}) \leq \min _{i} \frac{d_{i}}{b_{i i}}=\min _{i}\left\{\frac{d_{1}}{b_{11}}, \frac{d_{2}}{b_{22}}, \ldots, \frac{d_{n}}{b_{n n}}\right\} \triangleq \frac{d_{k}}{b_{k k}} \triangleq \rho .
$$

That is, the cost function in (4) is upper bounded by $\rho$.

The upper bound $\rho$ may be reachable. Consequently, the algorithm to design $\mathbf{F}$ is as follows. We first try to find a precoder $\mathbf{F}_{\rho} \in S$ with maximum power (i.e., satisfies (1) with equality) and which reaches the upper bound $\rho$ :

$$
\mu\left(\mathbf{F}_{\rho}\right)=\rho .
$$

Any of the three approaches in Section 4 can be used for this search. If successful, we have found an optimal solution to (4) and are done; set $\mathbf{F}$ equal to $\mathbf{F}_{\rho}$. If unsuccessful, we get F by implementing a fix for the approach used to search for $\mathbf{F}_{\rho}$. This fix is simply lowering the power of the $k$ th antenna and is explained in Section 5. The low complexity of the three approaches in Section 4 is due to Section 3 which reveals necessary structure and conditions for $\mathbf{U}_{\rho}=\mathbf{F}_{\rho} \mathbf{F}_{\rho}^{*}$.

\section{Necessary Structure and Conditions}

If $\mathbf{F}_{\rho}$ exists, the transmit covariance matrix $\mathbf{U}_{\rho}$ would have a special structure and certain conditions would be true. Assume $\mathbf{F}_{\rho}$ exists. Then, observing from (6), $\mathbf{e}_{k}$ must be an eigenvector of $\mathbf{A U}_{\rho}$ paired with the eigenvalue $\rho$, that is,

$$
\mathrm{AU}_{\rho} \mathbf{e}_{k}=\rho \mathbf{e}_{k} \Leftrightarrow \mathbf{U}_{\rho} \mathbf{e}_{k}=\rho \mathbf{A}^{-1} \mathbf{e}_{k}=\rho \mathbf{B} \mathbf{e}_{k}
$$


In particular, the $k$ th column of $\mathbf{U}_{\rho}$ must equal the $k$ thcolumn of $\mathbf{B}$ multiplied by $\rho$. Consequently, if $1<k<n$ and $\left[\begin{array}{lll}\mathbf{b}_{1}^{T} & b_{k k} & \mathbf{b}_{2}^{T}\end{array}\right]^{T}$ denotes the $k$ th column of $\mathbf{B}$, then, necessarily,

$$
\begin{aligned}
& \mathbf{U}_{\rho}=\mathbf{F}_{\rho} \mathbf{F}_{\rho}^{*}=\left[\begin{array}{ccc}
\boldsymbol{\Sigma}_{1} & \rho \mathbf{b}_{1} & \mathbf{L} \\
\rho \mathbf{b}_{1}^{*} & d_{k} & \rho \mathbf{b}_{2}^{*} \\
\mathbf{L}^{*} & \rho \mathbf{b}_{2} & \boldsymbol{\Sigma}_{2}
\end{array}\right]>\mathbf{0}, \\
& \mathbf{I}_{k-1} \circ \boldsymbol{\Sigma}_{1}=\operatorname{diag}\left(\left[\begin{array}{lll}
d_{1} & \cdots & d_{k-1}
\end{array}\right]\right) \text {, } \\
& \mathbf{I}_{n-k} \circ \boldsymbol{\Sigma}_{2}=\operatorname{diag}\left(\left[\begin{array}{lll}
d_{k+1} & \cdots & d_{n}
\end{array}\right]\right) \text {. }
\end{aligned}
$$

For the sake of clarity, the cases when $k=1$ or $n$ are omitted in this paper.

3.1. Necessary Conditions for $\mathbf{U}_{\rho}$ (1st Representation). As to be expected, the remaining unspecified elements of $\mathbf{U}_{\rho}$ (i.e., $\mathbf{L}$ and the off diagonal elements of $\boldsymbol{\Sigma}_{1}$ and $\boldsymbol{\Sigma}_{2}$ ) are not arbitrary. By reducing $\mathbf{U}_{\rho}$ to direct sum form using elementary block row and column operations, it can be seen that $\mathbf{U}_{\rho}>\mathbf{0}$ if and only if

$$
\begin{aligned}
& \boldsymbol{\Sigma}_{1}>\mathbf{0}, \quad \boldsymbol{\Sigma}_{2}>\mathbf{0}, \quad \boldsymbol{\Sigma}_{2}-\mathbf{L}^{*} \Sigma_{1}^{-1} \mathbf{L}>\mathbf{0}, \\
& \frac{b_{k k}^{2}}{d_{k}}>\mathbf{b}_{1}^{*} \boldsymbol{\Sigma}_{1}^{-1} \mathbf{b}_{1} \\
&+\left(\mathbf{b}_{2}-\mathbf{L}^{*} \boldsymbol{\Sigma}_{1}^{-1} \mathbf{b}_{1}\right)^{*}\left(\boldsymbol{\Sigma}_{2}-\mathbf{L}^{*} \boldsymbol{\Sigma}_{1}^{-1} \mathbf{L}\right)^{-1}\left(\mathbf{b}_{2}-\mathbf{L}^{*} \boldsymbol{\Sigma}_{1}^{-1} \mathbf{b}_{1}\right) .
\end{aligned}
$$

Since $\boldsymbol{\Sigma}_{2}-\mathbf{L}^{*} \boldsymbol{\Sigma}_{1}^{-1} \mathbf{L}>\mathbf{0}$ (see (12)), the following necessary condition can be derived from (13):

$$
\frac{b_{k k}^{2}}{d_{k}}>\mathbf{b}_{1}^{*} \Sigma_{1}^{-1} \mathbf{b}_{1} .
$$

Furthermore, since $\boldsymbol{\Sigma}_{1}>\mathbf{0}$, a redundant though useful necessary condition can be derived for the antenna powers $\left\{d_{1}, \ldots, d_{k}\right\}$, which depends only on the known parameters $\mathbf{b}_{1}$, and $b_{k k}$ :

$$
\frac{b_{k k}^{2}}{d_{k}}>\frac{\mathbf{b}_{1}^{*} \mathbf{b}_{1}}{\lambda_{\max }\left(\boldsymbol{\Sigma}_{1}\right)} \geq \frac{\mathbf{b}_{1}^{*} \mathbf{b}_{1}}{\operatorname{tr}\left(\boldsymbol{\Sigma}_{1}\right)}=\frac{\mathbf{b}_{1}^{*} \mathbf{b}_{1}}{d_{1}+d_{2}+\cdots+d_{k-1}} .
$$

An alternate necessary and sufficient condition for $\mathbf{U}_{\rho}>$ $\mathbf{0}$ can be given by simply interchanging the subscripts 1 and 2 , and $\mathbf{L}^{*}$ and $\mathbf{L}$ in (12), (13). Thus, one can easily show that

$$
\begin{gathered}
\frac{b_{k k}^{2}}{d_{k}}>\mathbf{b}_{2}^{*} \Sigma_{2}^{-1} \mathbf{b}_{2}, \\
\frac{b_{k k}^{2}}{d_{k}}>\frac{\mathbf{b}_{2}^{*} \mathbf{b}_{2}}{d_{k+1}+d_{k+2}+\cdots+d_{n}}
\end{gathered}
$$

are also necessary.

3.2. Necessary Conditions for $\mathbf{U}_{\rho}$ (2nd Representation). Use an appropriate invertible, symmetric, real $n \times n$ permutation matrix $\mathbf{P}$ to permute $d_{k}$ to the upper left corner:

$$
\mathbf{P}^{*} \mathbf{U}_{\rho} \mathbf{P}=\left[\begin{array}{cc}
d_{k} & \rho \widetilde{\mathbf{b}}^{*} \\
\rho \widetilde{\mathbf{b}} & \mathbf{Q}
\end{array}\right]
$$

(The structures of $\mathbf{P}, \mathbf{Q}$, and $\tilde{\mathbf{b}}$, are omitted here). It turns out that $\mathbf{U}_{\rho}>\mathbf{0}$ if and only if $\mathbf{P}^{*} \mathbf{U}_{\rho} \mathbf{P}>\mathbf{0}$. Thus, $\mathbf{U}_{\rho}>\mathbf{0}$ if and only if (noting that $d_{k}>0$ given in (1))

$$
\mathbf{Q}-\frac{d_{k}}{b_{k k}^{2}} \tilde{\mathbf{b}}^{*}>\mathbf{0} .
$$

Since both (12)-(13), and (18) are necessary and sufficient for $\mathbf{U}_{\rho}>\mathbf{0}$, (12)-(13) and (18) are equivalent to each other. Conveniently, the diagonal elements of $\mathbf{Q}, q_{i i}, i=1, \ldots, n-$ 1 , are just the diagonal elements of $\boldsymbol{\Sigma}_{1}, \boldsymbol{\Sigma}_{2}$ permutated. Consequently, requiring that the $q_{i i}, i=1, \ldots, n-1$, be equal to the correct antenna powers is equivalent to requiring conditions (11). Moreover, a redundant though useful necessary condition can easily be derived from (18) which only uses the antenna powers, $\widetilde{\mathbf{b}}$, and $b_{k k}$ :

$$
q_{i i}-\frac{d_{k}}{b_{k k}^{2}}\left|\tilde{b}_{i}\right|^{2}>0, \quad(i=1, \ldots, n-1) .
$$

\section{Three Approaches for Obtaining $U_{\rho}$}

Each of the following three approaches seeks to find an $\mathbf{U}_{\rho}$ (i.e., $\mathbf{L}, \boldsymbol{\Sigma}_{1}$, and $\boldsymbol{\Sigma}_{2}$ in (10) or equivalently $\mathbf{Q}$ in (17)) which satisfies the necessary structure and conditions in Section 3.

4.1. Rank 0 Approach (ROA). In this simple approach, the matrix $\mathbf{L}$ is chosen to have rank 0 (hence the name of the approach), that is, equal to all zeros. $\boldsymbol{\Sigma}_{1}$ and $\boldsymbol{\Sigma}_{2}$ are chosen to be diagonal matrices with the diagonal entries $\left(d_{1}, \ldots, d_{k-1}\right)$ and $\left(d_{k+1}, \ldots, d_{n}\right)$, respectively. Such a choice for $\mathbf{L}, \boldsymbol{\Sigma}_{1}$, and $\Sigma_{2}$ automatically satisfies (11), (12). If the last remaining necessary condition (13) is satisfied, construct $\mathbf{U}_{\rho}$ using (10) and check whether $\rho$ is the minimum eigenvalue of $\mathbf{A U}_{\rho}$. If both of these conditions are satisfied, decompose $\mathbf{U}_{\rho}$ to get a $\mathbf{F}_{\rho}$ (and a corresponding $\mathbf{G}$ ) and an optimum solution has been found. If either condition fails, use the fix in Section 5 . It is interesting to note that the decomposition from $\mathbf{U}_{\rho}$ to $\mathbf{F}_{\rho}$ is not unique; indeed, using $\mathbf{F}_{\rho}$ right multiplied by a unitary matrix is also a valid decomposition.

4.2. Rank 1 Approach (R1A). Choose $\boldsymbol{\Sigma}_{1}$ and $\boldsymbol{\Sigma}_{2}$ as in R0A. If $\mathbf{b}_{1}^{*} \boldsymbol{\Sigma}_{1}^{-1} \mathbf{b}_{1}=\mathbf{b}_{2}^{*} \boldsymbol{\Sigma}_{2}^{-1} \mathbf{b}_{2}$, use another approach. If $\mathbf{b}_{1}^{*} \boldsymbol{\Sigma}_{1}^{-1} \mathbf{b}_{1}>$ $\mathbf{b}_{2}^{*} \boldsymbol{\Sigma}_{2}^{-1} \mathbf{b}_{2}$, choose a rank one choice of $\mathbf{L}$,

$$
\mathbf{L}=\frac{\mathbf{b}_{1} \mathbf{b}_{2}^{*}}{\mathbf{b}_{1}^{*} \Sigma_{1}^{-1} \mathbf{b}_{1}} .
$$

This $\mathbf{L}$ makes the right-hand side of (13) as small as possible and independent of $\boldsymbol{\Sigma}_{2}$. If $\mathbf{b}_{1}^{*} \boldsymbol{\Sigma}_{1}^{-1} \mathbf{b}_{1}<\mathbf{b}_{2}^{*} \boldsymbol{\Sigma}_{2}^{-1} \mathbf{b}_{2}$, choose an alternative rank one choice,

$$
\mathbf{L}=\frac{\left(\mathbf{b}_{1} \mathbf{b}_{2}^{*}\right)}{\left(\mathbf{b}_{2}^{*} \Sigma_{2}^{-1} \mathbf{b}_{2}\right)} .
$$

With these $\boldsymbol{\Sigma}_{1}, \boldsymbol{\Sigma}_{2}$, and $\mathbf{L}$, the power constraints in (11) and the first two conditions of (12) are automatically satisfied. Furthermore, the condition $\boldsymbol{\Sigma}_{2}-\mathbf{L}^{*} \boldsymbol{\Sigma}_{1}^{-1} \mathbf{L}>\mathbf{0}$ in (12) is also satisfied-use the fact that $\mathbf{b}_{1}^{*} \boldsymbol{\Sigma}_{1}^{-1} \mathbf{b}_{1}>\mathbf{b}_{2}^{*} \boldsymbol{\Sigma}_{2}^{-1} \mathbf{b}_{2}$ when (20) 
is used and that $\mathbf{b}_{1}^{*} \boldsymbol{\Sigma}_{1}^{-1} \mathbf{b}_{1}<\mathbf{b}_{2}^{*} \boldsymbol{\Sigma}_{2}^{-1} \mathbf{b}_{2}$ when (21) is used. However, (13) needs to be checked. In addition, whether $\rho$ is the minimum eigenvalue of $\mathbf{A U}_{\rho}$ also needs to be checked. Same as in $R 0 A$, if both conditions are satisfied, decompose $\mathbf{U}_{\rho}$ to get a $\mathbf{F}_{\rho}$ (and a corresponding $\mathbf{G}$ ) and an optimum solution has been found. If either condition fails, use the fix in Section 5.

4.3. Permutation Approach (PA). This third approach, unlike the previous two, searches for $\mathbf{U}_{\rho}$ using the 2nd representation of necessary conditions (Section 3.2). It is based on two facts. The first is that $\mathbf{Q}=\mathbf{Q}^{*}$ and

$$
\lambda_{\min }(\mathbf{Q})>\eta_{\min } \triangleq d_{k} b_{k k}^{-2} \tilde{\mathbf{b}}^{*} \tilde{\mathbf{b}}
$$

together imply (18). The second is that a Hermitian Q satisfying (22) and having the correct diagonal entries (the antenna powers permutated) exists if and only if $\eta_{\min }<d_{i}$, for all $i$ (just apply the Schur-Horn Theorem [8]).

Granted that $\eta_{\min }<d_{i}$, for all $i$, the approach is as follows. First choose some $\varepsilon>0$ such that $\eta_{\min }+\varepsilon \equiv \gamma \leq d_{i}$, for all $i$. Next, find a Hermitian $\mathbf{Q}$ whose diagonal entries are the correct antenna powers, $\left\{q_{i i}\right\}$, and whose eigenvalues are lower bounded by $\gamma$. Once a $\mathbf{Q}$ is found, construct (in light of (17))

$$
\mathbf{U}_{\rho}=\left(\mathbf{P}^{*}\right)^{-1}\left[\begin{array}{cc}
d_{k} & \rho \widetilde{\mathbf{b}}^{*} \\
\rho \tilde{\mathbf{b}} & \mathbf{Q}
\end{array}\right] \mathbf{P}^{-1}
$$

If $\rho$ is the minimum eigenvalue of $\mathbf{A U}_{\rho}$, obtain $\mathbf{F}_{\rho}$ and a corresponding $\mathbf{G}$ from $\mathbf{U}_{\rho}$ as in $R 0 A$ and $R 1 A$; an optimum solution has been found. If there is a smaller eigenvalue than $\rho$, use the fix in Section 5.

There are various ways to find a Hermitian $\mathbf{Q}$ with diagonal entries $\left\{q_{i i}\right\}$ and eigenvalues lower bounded by $\gamma$. A closed form solution is shown in Appendix A and a projection approach is shown in Appendix B.

\section{A Fix}

Consider a L, $\boldsymbol{\Sigma}_{1}$, and $\boldsymbol{\Sigma}_{2}$ which satisfies (11) and (12). If (13) does not hold and/or if $\rho=d_{k} / b_{k k}$ is greater than the smallest eigenvalue of $\mathbf{A U}_{\rho}$, proceed as following. First, temporarily change the per-antenna power constraint for the $k$ th antenna by replacing $d_{k}$ by $d_{k}^{\prime}$. This makes $\rho$ become $d_{k}^{\prime} / b_{k k}, \mathbf{U}_{\rho}$ become

$$
\left[\begin{array}{ccc}
\boldsymbol{\Sigma}_{1} & \rho \mathbf{b}_{1} & \mathbf{L} \\
\rho \mathbf{b}_{1}^{*} & d_{k}^{\prime} & \rho \mathbf{b}_{2}^{*} \\
\mathbf{L}^{*} & \rho \mathbf{b}_{2} & \boldsymbol{\Sigma}_{2}
\end{array}\right],
$$

and so forth. Second, lower $d_{k}^{\prime}$ (maintaining $d_{k}^{\prime}>0$ ) until (13) holds. Then, continue lowering $d_{k}^{\prime}$ (maintaining $d_{k}^{\prime}>$ 0 ) and thus $\rho=d_{k}^{\prime} / b_{k k}$ until $\rho$ is the smallest eigenvalue of $\mathbf{A U}_{\rho}$. As Appendix C shows, one can always lower $d_{k}^{\prime}$ until this happens. Using this fix, (8) is thus obtained-granted, for a lower power constraint.

Lastly, now that $d_{k}^{\prime}$ is low enough, decompose $\mathbf{U}_{\rho}$ to get $\mathbf{F}_{\rho}$ (and a corresponding $\mathbf{G}$ ). This $\mathbf{F}_{\rho}$ is full rank since (1213) hold. In addition, it satisfies the inequality per-antenna power constraint (1) with the true $d_{k}$ and the $d_{i}$, for all $i \neq k$. Indeed,

$$
\mathbf{e}_{k}^{*}\left(\mathbf{F}_{\rho} \mathbf{F}_{\rho}^{*}\right) \mathbf{e}_{k}=d_{k}{ }^{\prime}<d_{k} .
$$

Thus, set $\mathbf{F}$ equal to $\mathbf{F}_{\rho}$.

Lowering $d_{k}^{\prime}$ until (13) holds is understandable-F needs to be full rank. But, why lower it to satisfy (8) for a lower power constraint? Instead of lowering $d_{k}^{\prime}$ further, decomposing $\mathbf{U}_{\rho}$ to get $\mathbf{F}$ at that point would yield a legitimate precoder. So, why continue to lower $d_{k}^{\prime}$ ? The reason is that it is observed numerically that continuing to lower $d_{k}^{\prime}$ actually raises the minimum eigenvalue of $\mathbf{A U}_{\rho}$. See Figure 6 and the discussion for it.

\section{Numerical Results}

The numerical results are divided into two parts. In the first part, two examples are given to demonstrate the proposed approaches of Section 4 and the fix of Section 5. In the second part, Monte Carlo simulations are used to investigate how suboptimal, if at all, is the proposed design methodology for $\mathbf{F}$. It also investigates how often the fix is needed.

6.1. Demonstration of the Proposed Design Methodology for F. Two examples are given here, each of which corresponds to one $\mathbf{H}$ and $\mathbf{R}_{\mathbf{n n}}$ (i.e., one $\mathbf{A}$ ). Without loss of generality, consider 10 antennas $(n=10)$ with identical power constraints $\left(d_{i}=10, i=1, \ldots, n\right)$. Thus, maximum allowable total power $P=\sum_{i=1}^{n} d_{i}=100$.

For the first numerical example, (8) is achieved by the $R 0 A, R 1 A$, and $P A$. The resulting eigenvalues of $\mathbf{A U}$ for each of them are plotted in Figure 1. The upper bound $\rho$ and the optimum result under the total power constraint (see (5)) are also plotted as benchmarks. Several interesting observations can be made. Firstly, since $P=\sum_{i=1}^{n} d_{i}$, the optimum solution for the total power case always is at least as good as that of the per-antenna case. Indeed, in Figure 1, the $\lambda_{1}(\mathbf{A U})$ (i.e., $\left.\lambda_{\min }(\mathbf{A U})\right)$ for the total power case is greater than $\rho$, an upper bound for the $\lambda_{1}(\mathbf{A U})$ 's of the per-antenna case. Secondly, the $R 0 A, R 1 A$, and $P A$ all result in optimum F's here; $\lambda_{1}(\mathrm{AU})$ for each of the three approaches is numerically equal to $\rho$. Thirdly, the eigenvalues are all equal for the total power case (as is always the case. See [1]). However, the eigenvalues for any of the approaches subject to the perantenna constraint are clearly not all equal.

In the second numerical example, the $R 0 A$ 's solution does not satisfy (13). Thus, the fix in Section 5 is applied to it. The eigenvalues and the $\rho$ resulting from the fix (i.e., $d_{k}^{\prime} / b_{k k}$ ) are plotted in Figure 2. The original $\rho$ and the optimum result under the total power constraint $P=100$ are also plotted for reference. As it is supposed to be, $\lambda_{1}(\mathbf{A U})$ for the fixed solution is numerically equal to the lowered $\rho$. The $d_{k}{ }^{\prime}$ is approximately equal to 4.4138 .

6.2. Investigation into the Effectiveness of the Proposed Design Methodology for F. In this subsection, we use Monte Carlo simulation to assess the effectiveness of the proposed approach and to show how far our suboptimum solution is 


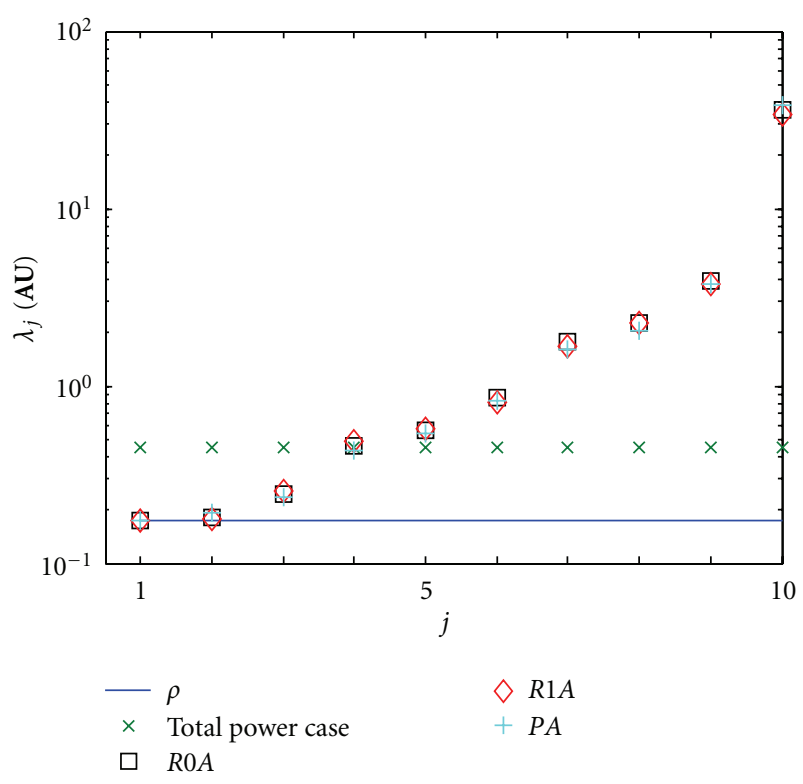

Figure 1: $\lambda_{j}(\mathrm{AU})$ as a function of $j$ (the index of eigenvalues) when the upper bound in (8) is reached.

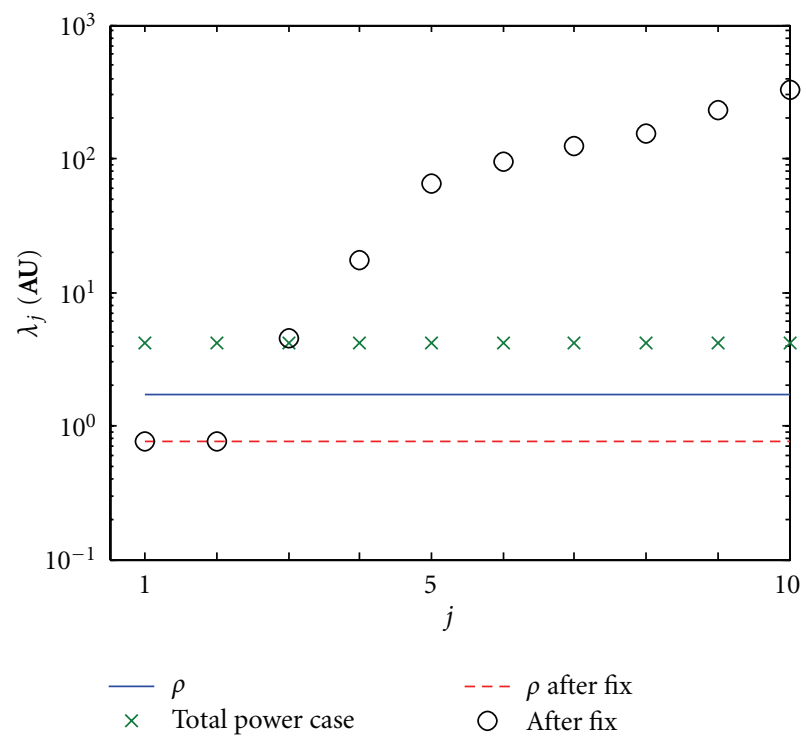

FIGURE 2: $\lambda_{j}(\mathrm{AU})$ as a function of $j$ (the index of eigenvalues) when the fix is used.

from the optimum solution. We did not prove that the upper bound in (8) is always achievable. How then do we get the optimum solution needed for this comparison? For a $\mathbf{R}_{\mathrm{n}}$, $P$, and $\mathbf{H}$, we obtain it by the following methodology. We do not specify the per-antenna power constraints $\left\{d_{i}\right\}$ at the beginning. Instead, we calculate the closed-form precoder in (5) and set its antenna powers as the $\left\{d_{i}\right\}$. In other words, if $\mathbf{F}_{o}$ denotes the precoder from (5), we set $\mathbf{e}_{i}^{*}\left(\mathbf{F}_{o} \mathbf{F}_{o}^{*}\right) \mathbf{e}_{i}$ as $d_{i}$, for all $i$. For this resulting problem, Appendix D proves that the precoder from (5) is an optimum solution to (4). Moreover, it achieves the upper bound in (8). With a $\mathbf{R}_{\mathbf{n n}}, \mathbf{H}$, and $\left\{d_{i}\right\}$ in hand where we know the upper bound $\rho$ is achievable, we can run our algorithm to get $\mathbf{F}$ and analyze its performance.

More specifically, this simulation is run as follows. The noise covariance matrix $\mathbf{R}_{\mathbf{n n}}$ is set equal to $\sigma^{2} \mathbf{I}$. The transmit signal-to-noise ratio (SNR) is defined as $=10 \log _{10}\left(P / \sigma^{2}\right)$. Transmit SNRs of $0,6,12$, and $18 \mathrm{~dB}$ are run. Both 4 and 8 antenna scenarios $(n=4,8)$ are run. For each transmit SNR and $n, 1000$ H's are randomly generated; elements of $\mathbf{H}$ are independent identically distributed $\mathrm{CN}(0,1)$ random variables. After the $\left\{d_{i}\right\}$ are determined by the total power closed-form solution (5), the $R 0 A$ and, if necessary, the fix are run.

First, consider the 8 antennas case $(n=8)$. For all the transmit SNRs and all the randomly generated $\mathbf{H}$, the $R 0 A$ does not achieve the upper bound in (8) and the fix in Section 5 is employed. The top figure in Figure 3 shows the histogram of the ratio between the new $d_{k}^{\prime}$ (after the fix) and $d_{k}$ for the transmit SNR $6 \mathrm{~dB}$ case. Since $d_{k}^{\prime}$ is smaller than $d_{k}$, the total power of the proposed $\mathbf{F}$ is smaller than the maximum total power allowed, $P$. The bottom figure in Figure 3 thus shows the histogram of the ratio between the total power of the proposed $\mathbf{F}$ and $P$. The histograms for the other transmit SNRs are not shown since they are so similar to Figure 3. Recall that if the fix is used, the obtained cost function value is $d_{k}^{\prime} / b_{k k}$. Since the optimum solution obtains the cost function value of $\rho=d_{k} / b_{k k}$, the top histogram of Figure 3 also shows how suboptimal the proposed algorithm is.

The optimal solution in (5) is better than the proposed solution with respect to the cost function in (4). However, the proposed solution has a much larger mutual information than the optimal solution (see Figure 4). According to [1], the mutual information for a $\mathrm{F}$ is

$$
\log _{2}\left|\mathbf{I}+\mathbf{H F F}^{*} \mathbf{H}^{*} \mathbf{R}_{\mathbf{n n}}^{-1}\right|,
$$

when the MMSE decoder is used-as is done here. The reason for the observed larger mutual information is as follows. The optimum solution in (5) diagonalizes the equivalent channel matrix GHF and equalizes all eigenchannels so that the resulting SINRs for all data streams are the same and equal to $\rho$ (see Appendix D and [1]). But, for the proposed solution, the SINRs of all the data streams are not the same and, moreover, most of them are larger than $\rho$ (see Figure 5). The SINR for the $i$ th stream when $\mathbf{F}=\left[\begin{array}{lll}\mathbf{f}_{1} & \cdots & \mathbf{f}_{n}\end{array}\right]$ and $\mathbf{G}=\left[\begin{array}{lll}\mathbf{g}_{1}^{T} & \cdots & \mathbf{g}_{n}^{T}\end{array}\right]^{T}$ are used is simply

$$
\frac{\left|\mathbf{g}_{i} \mathbf{H f}_{i}\right|^{2}}{\mathbf{g}_{i} \mathbf{R}_{\mathbf{n n}} \mathbf{g}_{i}^{*}+\sum_{j \neq i}\left|\mathbf{g}_{i} \mathbf{H} \mathbf{f}_{j}\right|^{2}}
$$

Figure 5 shows the normalized SINRs for the 8 data streams of the proposed solution for a sampling of the channel realizations. The transmit SNR is $6 \mathrm{~dB}$. The figures for the other channel realizations and transmit SNRs are not shown since they are so similar to Figure 5. For a given channel realization, the normalization factor is the same for all 8 data streams. It is $\rho$, the SINR of every data stream when the optimum solution is used (see Appendix D). For the 

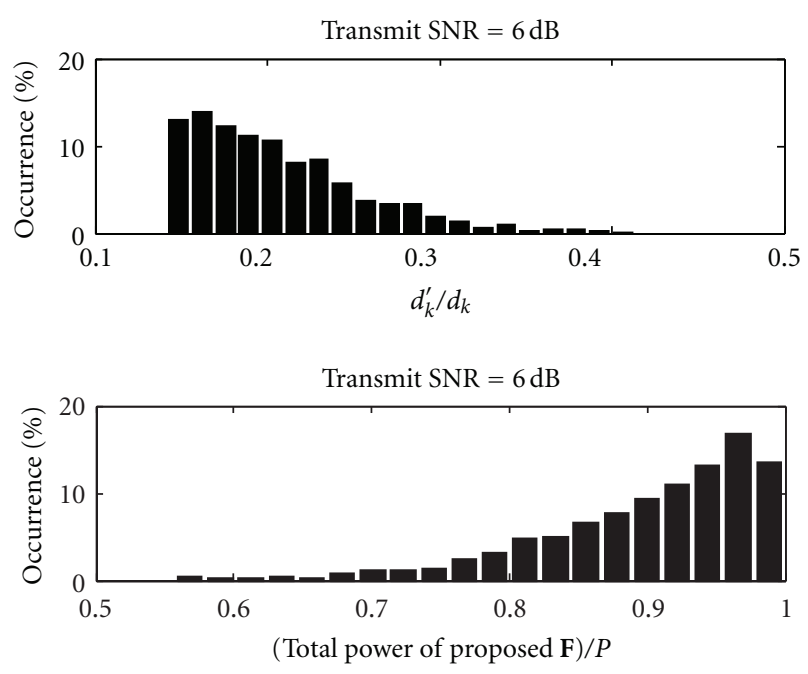

Figure 3: Eight-antenna example. Top: Histogram of the ratio between $d_{k}{ }^{\prime}$ (after the fix) and $d_{k}$. Bottom: histogram of the ratio between the total power of the proposed $\mathbf{F}$ and $P$.

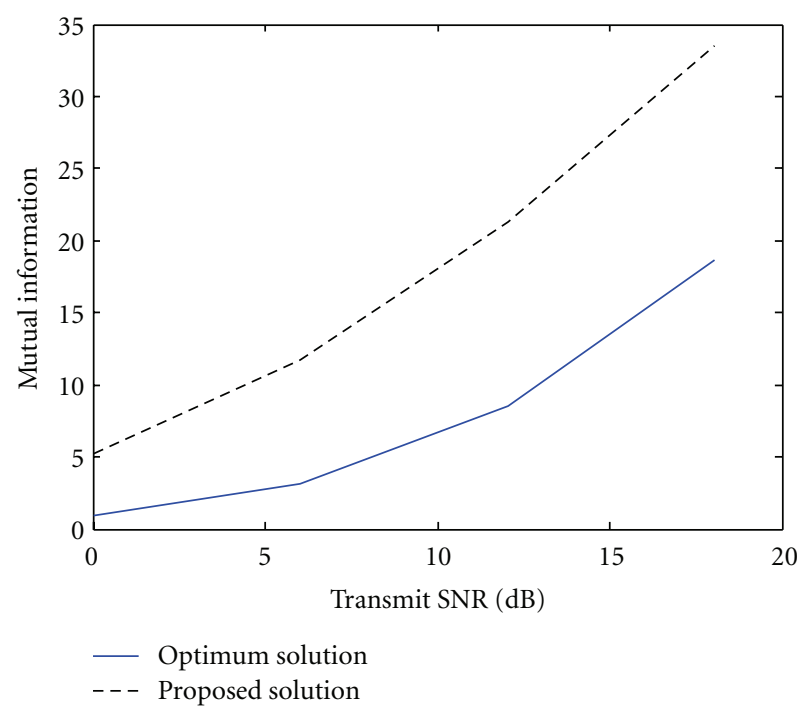

FIgURE 4: Eight-antenna example. Mutual information for the optimum precoder and the proposed precoder.

channel realizations shown in Figure 5, only one normalized SINR (out of 8 ) is less than 1 . Thus, only one data stream for the proposed solution has a lower SINR than the SINR of the 8 data streams for the optimal solution. This is also roughly the case for the other channel realizations as well.

Note that the optimal solution may have a smaller symbol error rate than the suboptimum solution if a ML receiver is used (according to the logic in Section 2.1). If the ML receiver is not employed, the optimal solution may not have any advantage over the proposed solution. For practical implementations, appropriate modulation and coding schemes can be selected to maximize the throughput when the precoder derived from the low complexity proposed algorithm is employed.

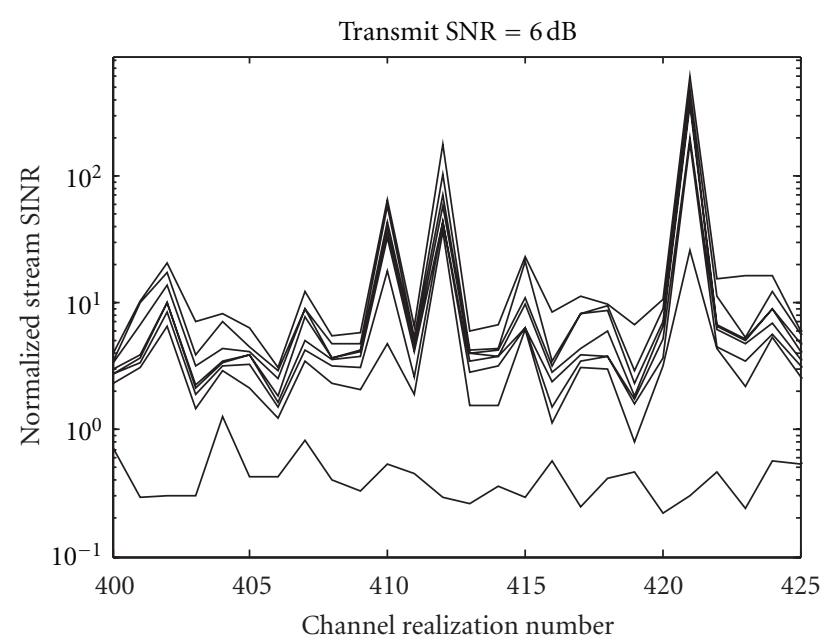

FIGURE 5: Eight-antenna example. Normalized SINRs for the 8 data streams for some channel realizations when the proposed algorithm is employed. The normalization factor is $\rho$, the SINR of every data stream when the optimum solution is used.

Section 5 said that numerical results showed that continuing to lower $d_{k}^{\prime}$ actually raises the minimum eigenvalue of $\mathbf{A U}_{\rho}$. Figure 6 shows a typical plot of the results of continuing to lower $d_{k}^{\prime}$ for a channel realization. At each iteration, $d_{k}^{\prime}$ is lowered by one percent. In the figure, $\rho=d_{k}^{\prime} / b_{k k}$ and $\mathbf{U}_{\rho}$ is given by (23) in accordance with the notation in Section 5. Indeed, the minimum eigenvalue of $\mathbf{A U}_{\rho}$ increases as $d_{k}^{\prime}$, and thus $\rho=d_{k}^{\prime} / b_{k k}$, is lowered. In fact, the increase is always monotonic (the value for an iteration is greater or equal to that of the previous iteration) in all the simulations save for one exception. In some channels (e.g., the one for Figure 6), $\lambda_{1}\left(\mathbf{A U}_{\rho}\right)$ for the last iteration may be smaller than $\lambda_{1}\left(\mathbf{A U}_{\rho}\right)$ for the second to last iteration. This may be due to overshooting since the step size for $d_{k}^{\prime}$ is fixed.

Next, consider the 4 antennas case $(n=4)$. Figure 7 shows the histogram of the ratio between $d_{k}^{\prime}$ (after the fix) and $d_{k}$. It also shows the histogram of the ratio between the total power of the proposed $\mathbf{F}$ and $P$. Figure 8 shows the mutual information for the optimum precoder and the proposed precoder. Figure 9 shows the proposed solution's normalized SINRs for the 4 data streams for some channel realizations. For a given channel realization, the normalization factor is $\rho$, the SINR of the 4 data streams when the optimum precoder in (5) is used.

Basically, the observations made for the eight-antenna example are still applicable here for the four-antenna example. Some notable differences and points are as follows. Firstly, the histogram of $d_{k}^{\prime} / d_{k}$ shifts to the right when going from $n=8$ to $n=4$. Secondly, the mutual information is smaller in the $n=4$ case. The transmit SNR gap between the two approaches is also smaller here as well. Lastly, for the $n=4$ case, the normalized SINRs suffer some degradation as transmit SNR increases. For the lower transmit SNRs, there are roughly 3 data streams with normalized SINRs above 1 and 1 data stream with normalized SINR below 1. For the higher transmit SNRs, there are roughly 2 data streams 


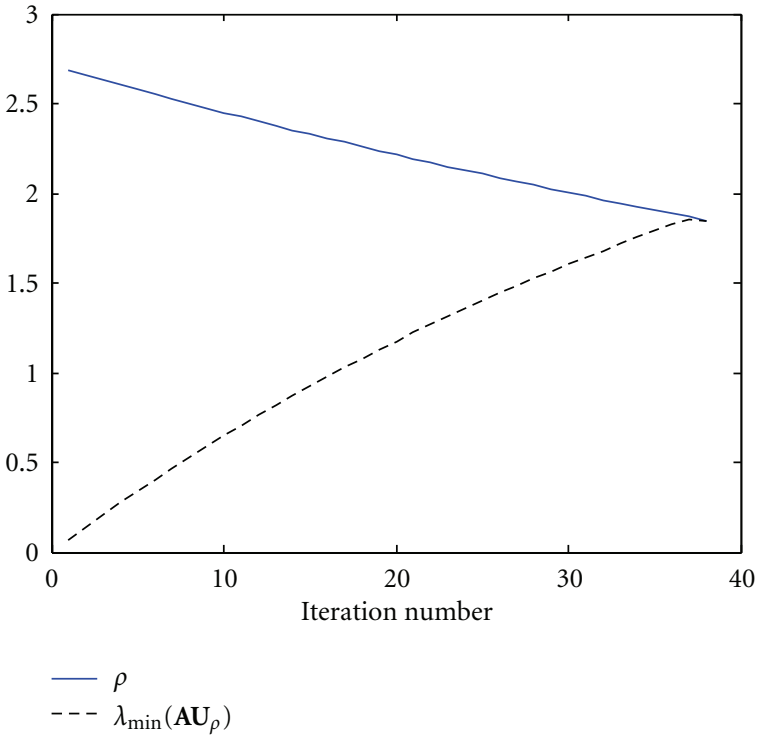

Figure 6: Eight-antenna example. Typical example of how the fix works.
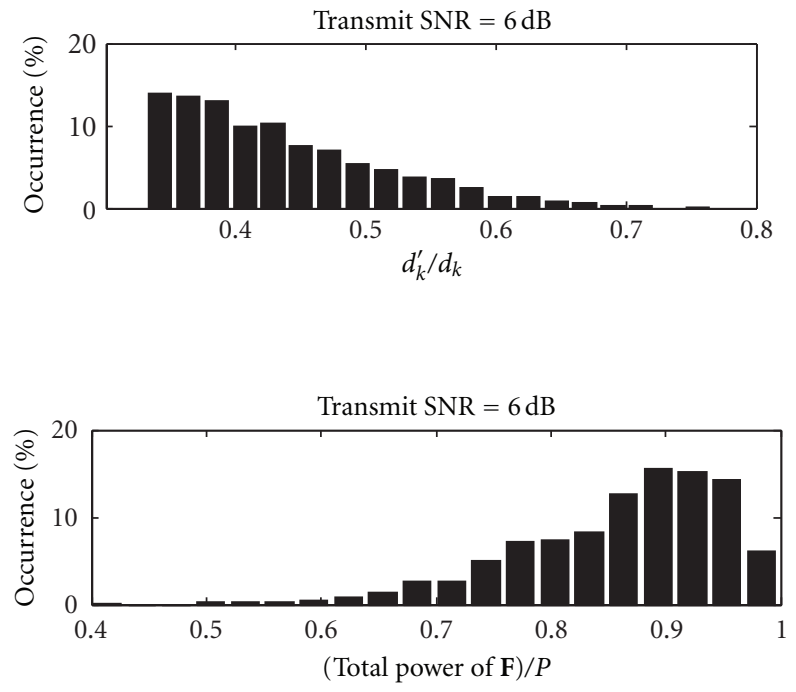

Figure 7: Four-antenna example. Top: histogram of the ratio between $d_{k}^{\prime}$ (after the fix) and $d_{k}$. Bottom: histogram of the ratio between the total power of the proposed $\mathbf{F}$ and $P$.

with normalized SINRs above 1 and 2 data streams with normalized SINRs below 1.

\section{Conclusion}

Considered here is the approximate minimum symbol error probability transceiver design subject to the practical perantenna power constraint. The metric to be maximized is a lower bound for the minimum distance of the symbol hypotheses. As in [1], the bound used is the minimum eigenvalue of a positive definite system matrix involving the precoder matrix, noise covariance matrix, and channel matrix. This max min problem is both interesting and challenging because the differentiation of the minimum

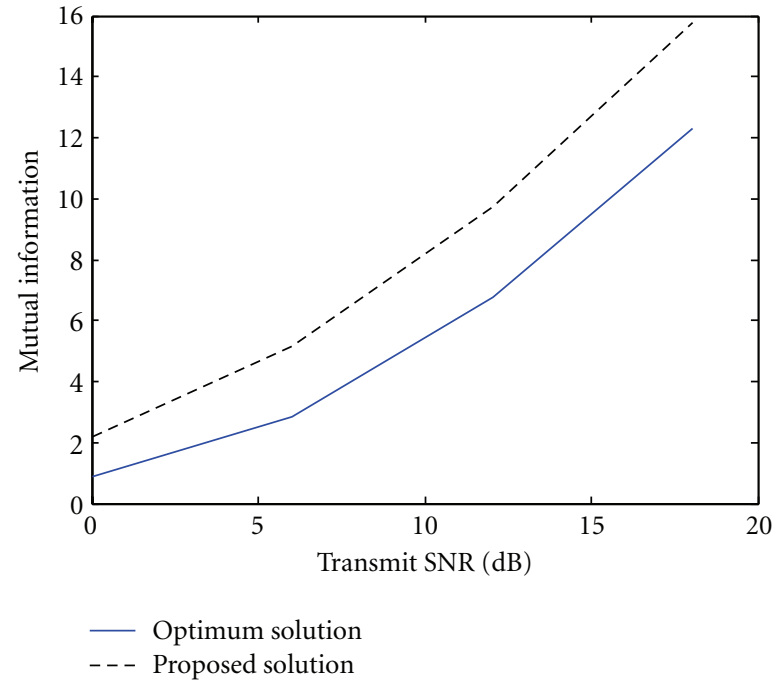

Figure 8: Four-antenna example. Mutual information for the optimum precoder and the proposed precoder.

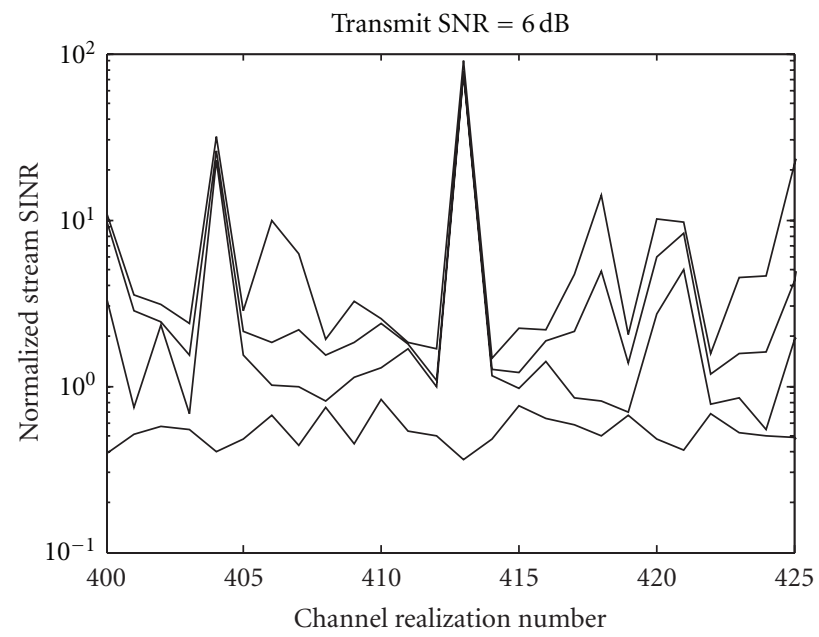

Figure 9: Four-antenna example. Normalized SINRs for the 4 data streams for some channel realizations when the proposed algorithm is employed. The normalization factor is $\rho$, the SINR of every data stream when the optimum solution is used.

eigenvalue cannot be performed explicitly. Remarkably, we are able to develop approaches to solve the design problem without using differentiation of eigenvalues or the popular method of Lagrange multipliers.

First, the upper bound for the cost function is established using the special structure of the system matrix and the power constraint. Then, necessary conditions and structures of the transmit covariance matrix for reaching the upper bound are obtained. Based on these necessary conditions and structures, three numerical approaches (rank zero, rank one and permutation) for obtaining the optimum precoder are developed. Since the upper bound is not always achieved, a possibly suboptimum fix is also given to be used, when necessary, after the proposed approaches.

In the total power constraint case, the eigenvalues of the optimum solution in [1] were always equal. Interestingly, the 
numerical results here show that this is not always the case for the per-antenna power constraint. Extensive numerical studies have been performed to assess the performances of the proposed methodology. Although the upper bound is not reached in most cases, good performances in mutual information and SINR are achieved. Moreover, the approach is very simple and can be of practical use.

\section{Appendices}

\section{A.}

Consider the $(n-1) \times(n-1)$ matrix $\mathbf{Y}=\gamma \mathbf{I}_{n-1}+\mathbf{X}$ where $\mathbf{X}$ $\geq \mathbf{0}$. For any $\mathbf{X} \geq \mathbf{0}, \mathbf{Y}$ is Hermitian and has eigenvalues lower bounded by $\gamma$. So, as long as

$$
\operatorname{diag}(\mathbf{X})=\left[q_{11}-\gamma, q_{22}-\gamma, \ldots, q_{n-1, n-1}-\gamma\right],
$$

$\mathbf{Y}$ is a valid choice of $\mathbf{Q}$.

To find a $\mathbf{X} \geq \mathbf{0}$ that satisfies (A.1) is not difficult. First, choose any $(n-1) \times(n-1)$ matrix $\mathbf{Z} \geq \mathbf{0}$ with positive diagonal entries $z_{i i}$. Then, form

$$
\mathbf{R} \equiv \operatorname{diag}\left(\left[\sqrt{\frac{q_{11}-\gamma}{z_{11}}}, \sqrt{\frac{q_{22}-\gamma}{z_{22}}}, \ldots, \sqrt{\frac{q_{n-1, n-1}-\gamma}{z_{n-1, n-1}}}\right]\right) .
$$

The product RZR is a valid choice of $\mathbf{X}$. Consequently, $\mathbf{Y}=\gamma$ $\mathbf{I}_{n-1}+\mathbf{R Z R}$ is a valid choice of $\mathbf{Q}$ and can be used in the $P A$.

\section{B.}

The projection approach finds a $\mathbf{Q}$ as follows. It begins by randomly generating a $(n-1) \times(n-1)$ unitary matrix $\mathbf{V}^{(0)}$ and $n-1$ real numbers, $\eta_{1} \geq \cdots \geq \eta_{n-1} \geq \gamma$. It then creates the initial guess at $\mathbf{Q}$ :

$$
\mathbf{Q}^{(0)}=\mathbf{V}^{(0)} \cdot \operatorname{diag}\left(\left[\eta_{1}, \ldots, \eta_{n-1}\right]\right) \cdot\left(\mathbf{V}^{(0)}\right)^{*}
$$

At the $j$ th iteration step $(j>1)$, it proceeds as follows.

(i) Force the diagonal elements of $\mathbf{Q}^{(j-1)}$ to be equal to $\left\{q_{i i}\right\}$.

(ii) Then, decompose $\mathbf{Q}^{(j-1)}$ as

$$
\mathbf{Q}^{(j-1)}=\mathbf{V}^{(j-1)} \cdot \operatorname{diag}\left(\left[\xi_{1}, \ldots, \xi_{n-1}\right]\right) \cdot\left(\mathbf{V}^{(j-1)}\right)^{*}
$$

to get the unitary matrix $\mathbf{V}^{(j-1)}$ and the real numbers $\xi_{1} \geq \cdots \geq \xi_{n-1}$.

(iii) If $\xi_{n-1} \geq \gamma$, set $\mathbf{Q}$ equal to $\mathbf{Q}^{(j-1)}$ and stop iterating. If not, let $\xi_{i}=\max \left(\xi_{i}, \gamma\right)$, for all $i$, and create

$$
\mathbf{Q}^{(j)}=\mathbf{V}^{(j-1)} \cdot \operatorname{diag}\left(\left[\xi_{1}, \ldots, \xi_{n-1}\right]\right) \cdot\left(\mathbf{V}^{(j-1)}\right)^{*}
$$

(iv) Move onto the $(j+1)$ th iteration step.
A $\mathbf{Q}$ is always found. That is, the above iteration always converges. The reason is as follows. The iteration simply projects between two closed, convex subsets of the Hilbert space $\mathbb{C}^{n \times n}$ :

$$
\begin{gathered}
\left\{\mathbf{T} \in \mathbb{C}^{n \times n} \mid \operatorname{diag}(\mathbf{T})=\left[q_{11} \cdots q_{n-1, n-1}\right], \mathbf{T}=\mathbf{T}^{*}\right\}, \\
\left\{\mathbf{V} \Theta \mathbf{V}^{*} \mid \mathbf{V}^{-1}=\mathbf{V}^{*} \in \mathbb{C}^{n \times n},\right. \\
\left.\Theta=\operatorname{diag}\left(\left[\theta_{1}, \ldots, \theta_{n}\right]\right) \in \mathbb{R}^{n \times n}, \theta_{i} \geq \gamma\right\}
\end{gathered}
$$

As the intersection of the two subsets is nonempty (by the Schur-Horn Theorem [8]), the iteration will converge [9].

C.

Assume that (10)-(13) hold but not (8). It will be proved here that (8) can be satisfied as well by simply lowering $d_{k}$ and thus $\rho$ (maintaining $d_{k}, \rho>0$ ). The proof can be split into four parts. The first part is to realize that (10) implies that $\rho$ is an eigenvalue of $\mathbf{A} \mathbf{U}_{\rho}$ (use (9)). The second part is to find a $(n-1) \times(n-1)$ matrix whose eigenvalues are precisely $\mathbf{A U}_{\rho}$ 's other $n-1$ eigenvalues. To this end, introduce the partition

$$
\mathbf{A}=\left[\begin{array}{lll}
\mathbf{C}_{11} & \mathbf{c}_{12} & \mathbf{C}_{13} \\
\mathbf{c}_{12}^{*} & \mathbf{c}_{22} & \mathbf{c}_{23} \\
\mathbf{C}_{13}^{*} & \mathbf{c}_{23}^{*} & \mathbf{C}_{33}
\end{array}\right]
$$

where $c_{22}$ is the $k k$ th element of $\mathbf{A}$. In addition, note that $\mathbf{A}$ times the $k$ th column of $\mathbf{U}_{\rho}$ is equal to $\rho \mathbf{e}_{k}$ due to $\mathbf{B}=\mathbf{A}^{-1}$. With some straightforward steps, it can thus be seen that

$$
\mathbf{M}\left(d_{k}\right)=\left[\begin{array}{ll}
\mathbf{C}_{11} & \mathbf{C}_{13} \\
\mathbf{C}_{13}^{*} & \mathbf{C}_{33}
\end{array}\right]\left[\begin{array}{cc}
\boldsymbol{\Sigma}_{1} & \mathbf{L} \\
\mathbf{L}^{*} & \mathbf{\Sigma}_{2}
\end{array}\right]+\frac{d_{k}}{b_{k k}}\left[\begin{array}{lll}
\mathbf{c}_{12} \mathbf{b}_{1}^{*} & \mathbf{c}_{12} \mathbf{b}_{2}^{*} \\
\mathbf{c}_{23}^{*} \mathbf{b}_{1}^{*} & \mathbf{c}_{23}^{*} \mathbf{b}_{2}^{*}
\end{array}\right]
$$

is a suitable candidate matrix. The third part is to realize that the eigenvalues of

$$
\left[\begin{array}{ll}
\mathbf{C}_{11} & \mathbf{C}_{13} \\
\mathbf{C}_{13}^{*} & \mathbf{C}_{33}
\end{array}\right]\left[\begin{array}{cc}
\Sigma_{1} & \mathbf{L} \\
\mathbf{L}^{*} & \mathbf{\Sigma}_{2}
\end{array}\right]
$$

the first term in $\mathbf{M}\left(d_{k}\right)$, are positive and independent of $d_{k}$. To this end, note that (C.3) is the product of two positive definite matrices. Then, note that the eigenvalues of such a product must be positive [10]. Finally, using the fact that "the eigenvalues of a square...complex matrix depend continuously upon its entries" ([11]: Appendix D) and a limiting argument, the last part is to realize that one can lower $d_{k}$ and thus $\rho$ (maintaining $d_{k}, \rho>0$ ) until all the eigenvalues of $\mathbf{M}\left(d_{k}\right)$ are $\geq \rho$.

\section{D.}

Let $\mathbf{F}_{o}$ denote the precoder from (5). As it is an optimal solution to the optimization problem in (5), $\operatorname{tr}\left\{\mathbf{F}_{o} \mathbf{F}_{o}^{*}\right\}=P$. But, what are its antenna powers? It turns out that the $i$ th antenna has power $b_{i i} P / \operatorname{tr}\left(\Lambda^{-1}\right)$ because

$$
\mathbf{F}_{o} \mathbf{F}_{o}^{*}=\mathbf{V} \Lambda^{-1 / 2} \Lambda^{-1 / 2} \mathbf{V}^{*}\left(\frac{P}{\operatorname{tr}\left(\Lambda^{-1}\right)}\right)=\mathbf{B}\left(\frac{P}{\operatorname{tr}\left(\Lambda^{-1}\right)}\right) .
$$


Here, we used the fact that $\mathbf{B}=\mathbf{A}^{-1}$ and $\mathbf{A}=\mathbf{V} \mathbf{\Lambda} \mathbf{V}^{*}$.

Let us say, for all $i$, we set $d_{i}$ equal to $\mathbf{F}_{o}$ 's $i$ th antenna power:

$$
d_{i}=b_{i i} \frac{P}{\operatorname{tr}\left(\Lambda^{-1}\right)} .
$$

Then from (7) and (D.2), we have the upper bound

$$
\rho=\frac{P}{\operatorname{tr}\left(\Lambda^{-1}\right)} .
$$

It turns out that $\mathbf{F}_{o}$ reaches this upper bound. Direct computation shows this. From (5), we have

$$
\begin{aligned}
\mathbf{F}_{o}^{*} \mathbf{A F}_{o} & =\Lambda^{-1 / 2} \mathbf{V}^{*} \mathbf{V} \mathbf{\Lambda} \mathbf{V}^{*} \mathbf{V} \boldsymbol{\Lambda}^{-1 / 2}\left(\frac{P}{\operatorname{tr}\left(\boldsymbol{\Lambda}^{-1}\right)}\right) \\
& =\mathbf{I}\left(\frac{P}{\operatorname{tr}\left(\boldsymbol{\Lambda}^{-1}\right)}\right) .
\end{aligned}
$$

From (D.3) and (D.4), we have

$$
\lambda_{\min }\left(\mathbf{F}_{o}^{*} \mathbf{A F}_{o}\right)=\left(\frac{P}{\operatorname{tr}\left(\Lambda^{-1}\right)}\right)=\rho .
$$

In summary, if the $d_{1}, \ldots, d_{n}$ of the per-antenna power constraint (1) are defined using the antenna powers of $\mathbf{F}_{o}, \mathbf{F}_{o}$ reaches the upper bound $\rho$ and is thus an optimum solution to $(4)$.

\section{Disclosure}

A part of this manuscript appears in Enoch Lu's dissertation, submitted to the Faculty of the Polytechnic Institute of New York University in partial fulfillment of the requirements for the degree Doctor of Philosophy (Electrical Engineering) January 2012.

\section{Acknowledgment}

The authors would like to express their gratitude to Professor Dante Youla for his comments.

\section{References}

[1] A. Scaglione, P. Stoica, S. Barbarossa, G. B. Giannakis, and H. Sampath, "Optimal designs for space-time linear precoders and decoders," IEEE Transactions on Signal Processing, vol. 50, no. 5, pp. 1051-1064, 2002.

[2] D. P. Palomar, J. M. Cioffi, and M. A. Lagunas, "Joint Tx-Rx beamforming design for multicarrier MIMO channels: a unified framework for convex optimization," IEEE Transactions on Signal Processing, vol. 51, no. 9, pp. 2381-2401, 2003.

[3] D. P. Palomar, "Unified framework for linear MIMO transceivers with shaping constraints," IEEE Communications Letters, vol. 8, no. 12, pp. 697-699, 2004.

[4] F. Rey, M. Lamarca, and G. Vazquez, "Robust power allocation algorithms for MIMO OFDM systems with imperfect CSI," IEEE Transactions on Signal Processing, vol. 53, no. 3, pp. 1070 1085, 2005.
[5] Y. K. Yazarel and D. Aktas, "Downlink beamforming under individual SINR and per antenna power constraints," in Proceedings of the IEEE Pacific Rim Conference on Communications, Computers and Signal Processing (PACRIM '07), pp. 422425, August 2007.

[6] C. C. Weng and P. P. Vaidyanathan, "Per-antenna power constrained MIMO transceivers optimized for BER," in Proceedings of the 42nd Asilomar Conference on Signals, Systems and Computers (ASILOMAR '08), pp. 1300-1304, October 2008.

[7] M. C. H. Lim, M. Ghogho, and D. C. McLernon, "Reduced complexity design for weighted harmonic mean SINR maximization in the multiuser MIMO downlink," in Proceedings of the IEEE 10th Workshop on Signal Processing Advances in Wireless Communications (SPAWC '09), pp. 206-210, June 2009.

[8] M. T. Chu, "Constructing a Hermitian Matrix from Its Diagonal Entries and Eigenvalues," SIAM Journal on Matrix Analysis and Applications, vol. 16, pp. 207-217, 1995.

[9] H. Stark and Y. Yang, Vector Space Projections: A Numerical Approach to Signal and Image Processing, Neural Nets, and Optics, John Wiley \& Sons, 1998.

[10] S. Hu-yun, "Estimation of the eigenvalues of $A B$ for $A>0$, B >0," Linear Algebra and Its Applications, vol. 73, pp. 147-150, 1986.

[11] R. A. Horn and C. R. Johnson, Matrix Analysis, Cambridge University Press, New York, NY, USA, 1985. 

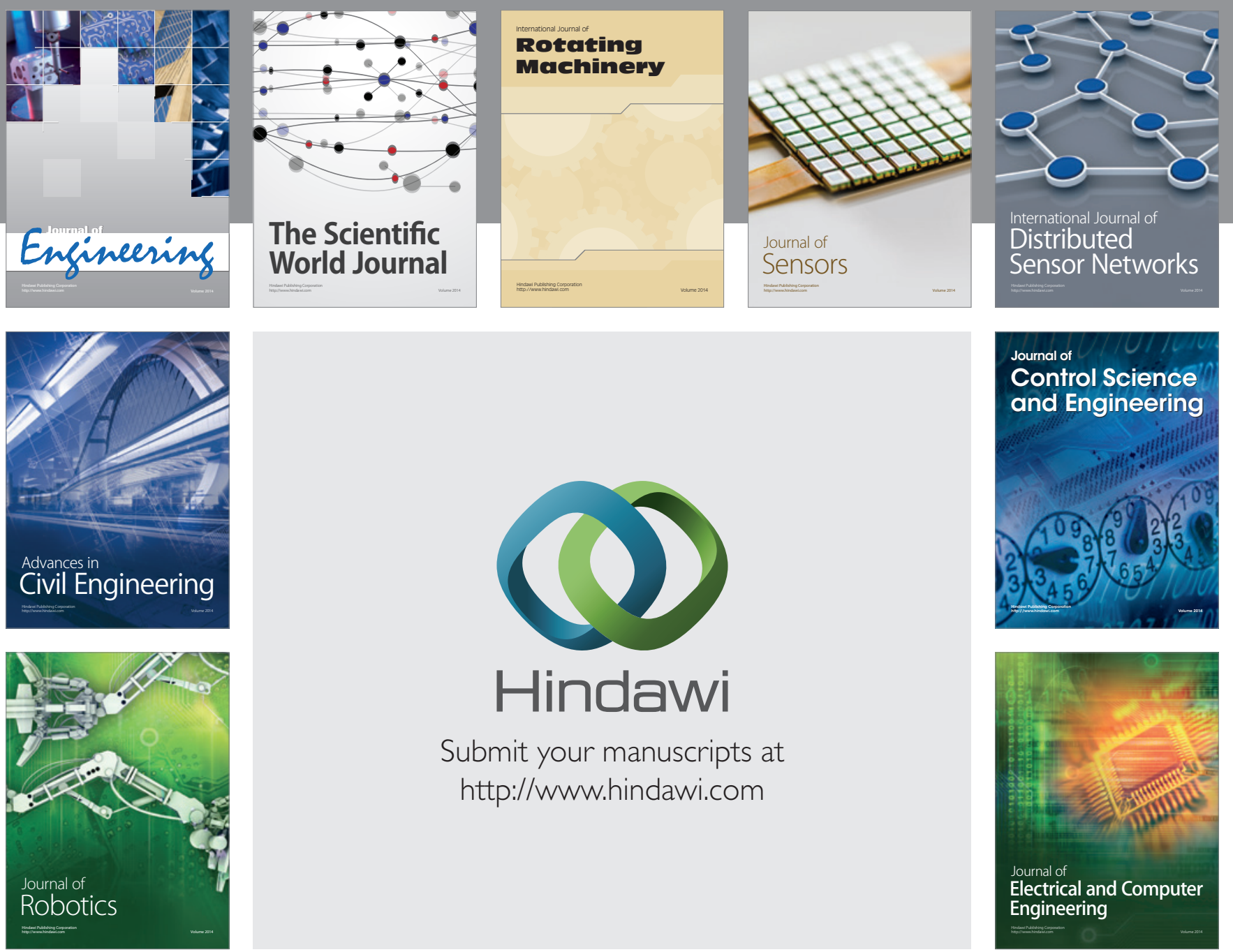

Submit your manuscripts at

http://www.hindawi.com
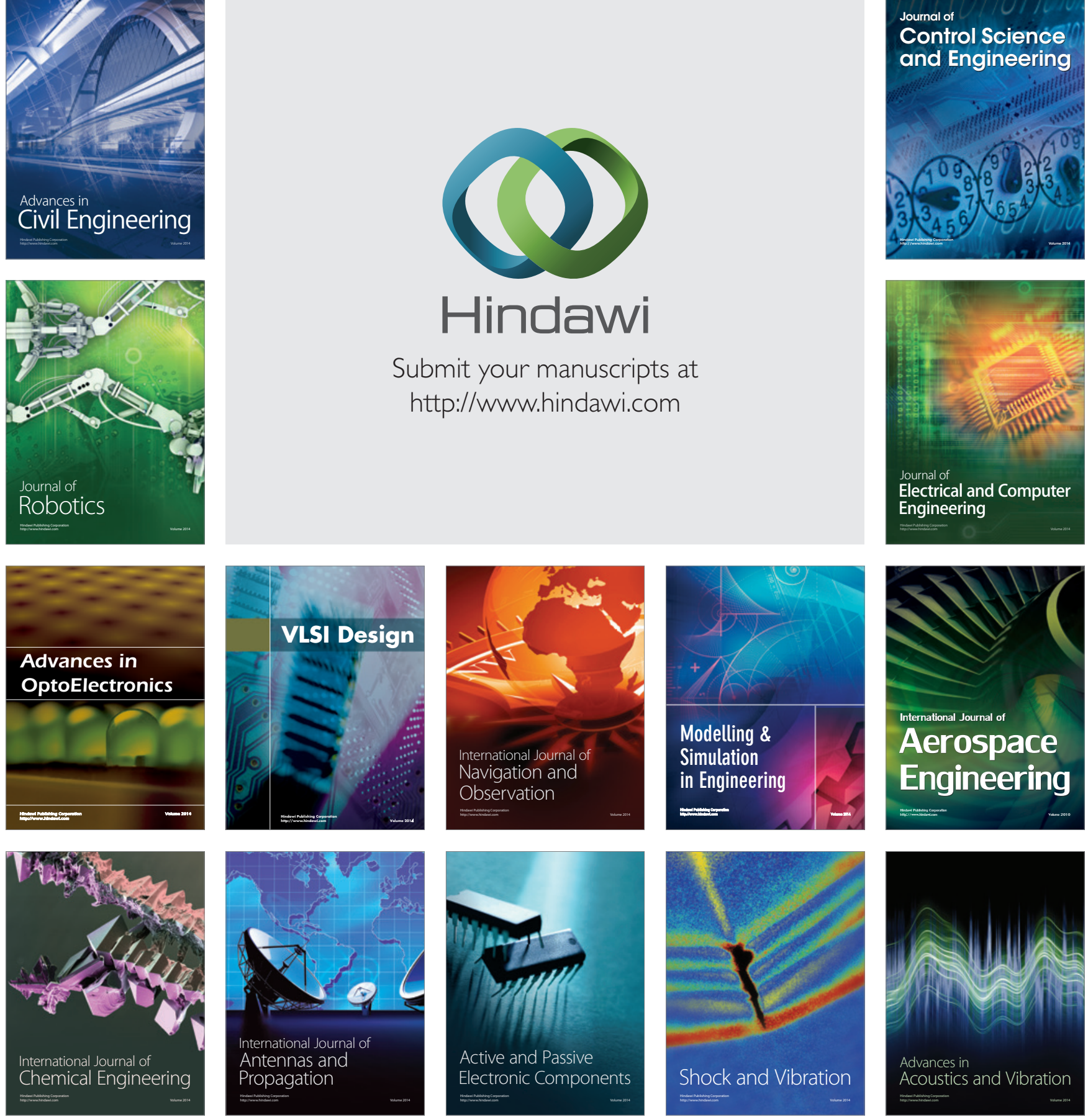